\title{
Effect of interfacial friction during forging of solid powder discs of large slenderness ratio
}

\author{
R K RANJAN $^{1}$ and S KUMAR ${ }^{2}$ \\ ${ }^{1}$ Applied Mechanics Department, and ${ }^{2}$ Production Engineering Department, Birla \\ Institute of Technology, Mesra, Ranchi 835 215, India \\ e-mail: rkranjanbit@yahoo.com
}

MS received 30 July 2003; revised 13 January 2004

\begin{abstract}
The paper reports an investigation into the effect of interfacial friction law during the forging of a powder circular disc with large slenderness ratio $(L / D)$, between two flat dies. The deformation pattern during the operation is influenced by many factors, which interact with each other in a complex manner. The relative velocity between the work piece material and the die surface, together with high interfacial pressure and/or deformation modes, creates the conditions essential for adhesion in addition to sliding. The decisive factors are the interfacial conditions, initial relative density of the preform and geometry of the preform. An attempt has been made to determine the most realistic interfacial friction law and die pressures developed during such forging using an upper bound approach. The results so obtained are presented graphically and discussed critically to illustrate the interaction of various interfacial friction laws involved.
\end{abstract}

Keywords. Slenderness ratio; preform; interfacial friction law.

\section{Introduction}

During the last few years metal-powder components have assumed an important position in industry, as they are being used successfully in a wide range of applications. Both the mechanical and metallurgical properties of the metal-powder components compare favourably with those of wrought materials (Ramakrishnan 1980). Bulk processing of metal-powder preforms is a convenient method of reducing or eliminating the porosity from conventional powder metallurgy products. The process is attractive because it avoids a large number of operations, high scrap losses and high-energy consumption associated with conventional manufacturing processes such as casting, machining etc. In this new technology, sintered porous powder-preforms are used as starting materials in metal forming processes. metalpowder products manufactured by this new technology are comparable and in some cases even superior to cast and wrought products.

The deformation pattern during the forging of metal-powder preforms is different from conventional wrought metal forging and the following characteristics of porous materials undergoing deformation must be taken into account:

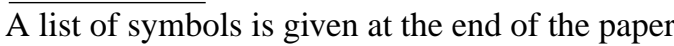




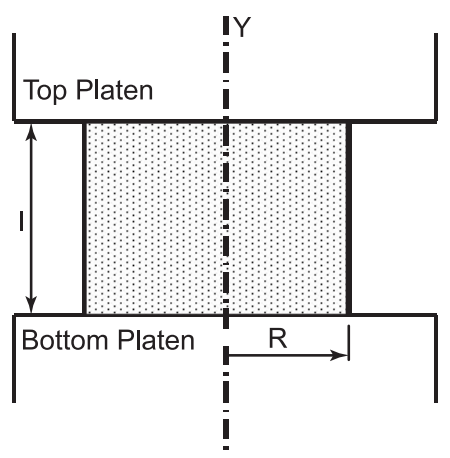

Figure 1. Forging assembly.

- Change in density occurs during plastic deformation;

- Yielding of porous metals is not completely insensitive to the hydrostatic stress imposed during processing.

Although a considerable amount of work has been reported recently as the various technological aspects of the industrial processing of metal-powder preforms (Sutradhar 1995, Jha \& Kumar 1983, 1996, 1997; Agrawal \& Kumar 1999; Kumar et al 2001), no systematic attempt has been made so far to study the effect of various interfacial friction laws and its effect on processing load and deformation characteristics during forging of circular discs of large slenderness ratio $(L / D)$.

This paper reports an investigation into the forging of circular preforms, with large values of slenderness ratio, between two flat dies (figure 1) considering various interfacial friction laws. In this forming operation, the nature of the flow of material is not completely understood and, for actual cases, an exact formulation of velocity model cannot be made. The main objective of this paper is to study the effect of interfacial friction laws and slenderness ratio of the specimen on the deformation pattern.

\section{Interfacial friction law}

Many solutions to problems in forging are obtained with constant shear factor, Coulomb friction and viscous friction assumed between die and preform. These theories are associated with forging practices, which prevailed more than 50 years ago. The basic shortcomings of these theories are the absence of a sufficient justification for the value of constant shear forces adopted in them. The correct determination of friction forces at the interface is of great practical importance.

It is well known that in plastic deformation, the surface of the preform is distorted and takes on an impression of the tool surface. Therefore, the actual contact area, as far as the specific cohesion of the contact surface is concerned, is not negligible as in the case of elastic deformation. Hence, friction in plastic deformation is essentially different from sliding friction in machine parts.

Direct metal-to-metal contact between the die and the preform is usually undesirable, since this contact results in welding and shearing of surface asperities and gives rise to high friction values and deterioration of the product. In practice, contact is prevented either by the presence of a surface oxide film as in many hot working processes or by the introduction of lubricant. In either case, however, high relative velocities between the preform and die surface combined 
with high interface pressure and/or deformation modes cause breakdown of the surface film and allow the new surface to come into contact with the die surface and facilitate the intimate contact essential for adhesion. The basic affinity of the die and the preform then determines the friction value. For such a case, the preform being forged does not necessarily slip along the surface of the dies. At the same time, it would also be erroneous to deny completely the existence of slip between preform and dies. For such a mechanism of composite friction, which usually occurs in axisymmetric processes, the shear equation becomes

$$
\tau=\mu\left[p_{a v}+\rho_{0} \phi_{0}\right]
$$

Friction conditions between deforming tool and work piece in metal forming are of the greatest importance with respect to a number of factors such as force and mode of deformation, properties of the finished specimen and resulting surface roughness. The relative velocity between the work piece material and the die surface together with high interfacial pressure and/or deformation modes create the conditions essential for adhesion in addition to sliding (Deryagin 1952).

The pattern of the metal flow during the forging of a metal-powder preform is such that there exists two zones, an inner one where no relative movement between work piece and die occurs (the sticking zone) and an outer zone where sliding occurs. Therefore, the appropriate friction laws for particular condition is:

$$
\tau=\mu\left[p_{a v}+\rho_{0} \phi_{0}\left\{1-\left(R_{m}-R\right) / n R_{0}\right\}\right],
$$

where $r$ denotes the sticking zone radius and which may be approximated by the relation given by Rooks (1974) and $n \gg 1$.

Considering the following interfacial laws, the expression for die load has been obtained during the forging of a powder preform

$$
\begin{aligned}
\text { (i) } \tau & =\mu p_{a v}, \\
\text { (ii) } \tau & =\mu\left[p_{a v}+\rho_{0} \phi_{0}\right] \\
\text { (iii) } \tau & =\mu\left[p_{a v}+\rho_{0} \phi_{0}\left(1-R / n R_{0}\right)\right] \\
\text { (iv) } \tau & =\mu\left[p_{a v}+\rho_{0} \phi_{0}\left(1-\left(R_{m}-R\right) / n R_{0}\right)\right] .
\end{aligned}
$$

\section{Yield criteria and plastic deformation of powder preforms}

In an investigation of the plastic deformation of sintered metal-powder preforms it is clear that change in volume occurs due to porosity. A preform of high density yields at a relatively high stress whereas a low density preform yields at a relatively low stress. Even hydrostatic stress can cause the sintered metal-powder preforms to yield, as the yield surface is closed on the hydrostatic stress axis. Also, density distribution does not seem to be uniform throughout, being high in the central region and low at the edges. The density distribution is more uniform for a smaller coefficient of friction and for a higher initial-density preform.

Tabata \& Masaki (1977) proposed the following yield criterion for porous metal-powder preforms:

$$
\rho^{k}=\sqrt{3 J_{2}^{\prime}} \pm 3 \eta \sigma_{m}
$$




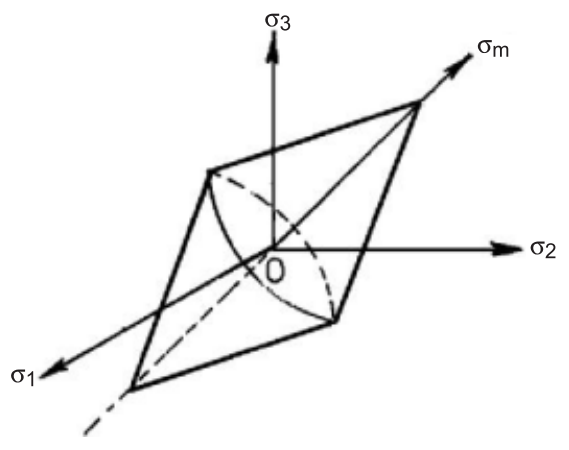

Figure 2. Yield surface for porous metal-powder preforms.

The negative sign is taken for $\sigma_{m} \leqslant 0$ and the positive sign is taken for $\sigma_{m}>0$.

Figure 2 shows that the yield surface for a porous metal-powder preform given by the above equation consists of two cones, the height of the cones increasing with increasing $\rho$. When $\rho=1$, i.e. a pore-free metal, the cone becomes a cylinder and the equation reduces to the Von Mises yield criterion.

The values of $\eta$ and $k$ were determined experimentally from simple compression and tension tests of sintered copper-powder preforms (Tabata \& Masaki 1977) as

$$
\begin{aligned}
& \eta=0.54(1-\rho)^{1.2}, \text { for } \sigma_{m} \leqslant 0, \\
& \eta=0.55(1-\rho)^{0.83}, \text { for } \sigma_{m}>0 \text { and } k=2 .
\end{aligned}
$$

For the axisymmetric condition the yield criterion reduces to (Tabata \& Masaki 1975)

$$
\sigma_{1}=\frac{\rho^{k} \sigma_{0}}{(1-2 \eta)}+\frac{(1+\eta)}{(1-2 \eta)} \sigma_{2} .
$$

\section{Velocity field and strain rate}

The velocity field for the case under consideration is

$$
\begin{aligned}
U_{R} & =[(1-2 \eta) U R /(1+\eta) L][1+a \sin (2 \pi y / \lambda)], \\
U_{y} & =U\left[(1-(2 y / L))+\frac{a}{N \pi}(\cos (2 \pi y / \lambda)-\cos 2 N \pi)\right] ; \quad U_{\theta}=0,
\end{aligned}
$$

where $a=-N \pi / 2$ for $N=L / \lambda=1 / 2,3 / 2, \ldots$.

The strain rates as calculated from (4) are

$$
\begin{aligned}
\varepsilon_{R} & =\frac{(1-2 \eta) U}{(1+\eta) L}\left[1+a \sin \frac{2 \pi y}{\lambda}\right], \quad \varepsilon_{y}=-\frac{2 U}{L}\left[1+a \sin \frac{2 \pi y}{\lambda}\right], \\
\varepsilon_{\theta} & =\frac{(1-2 \eta) U}{(1+\eta) L}\left[1+a \sin \frac{2 \pi y}{\lambda}\right], \quad \varepsilon_{y R}=-\frac{(1-2 \eta) U R \pi a}{(1+\eta) L \lambda} \cos \frac{2 \pi y}{\lambda}, \\
\varepsilon_{\theta R} & =\varepsilon_{\theta y}=0 .
\end{aligned}
$$

The relationship of volume change between the strain rates is given as (Tabata \& Masaki 1975)

$$
\varepsilon_{R}+[(1-2 \eta) / 2(1+\eta)] \varepsilon_{y}=0 .
$$




\section{Internal power of deformation}

The internal power of deformation is given by

$$
W_{i}=\frac{2}{\sqrt{3}} \sigma_{0} \int_{v}\left[\frac{1}{2}\left(\varepsilon_{R}^{2}+\varepsilon_{\theta}^{2}+\varepsilon_{y}^{2}\right)+2 \varepsilon_{R \theta}^{2}\right]^{1 / 2} \mathrm{~d} V
$$

where $\mathrm{d} V=2 \pi$ R.dR.dy.

Putting in the value of strain rates from (5), integration gives

$$
W_{i}=\frac{4 \pi(1-2 \eta) U \sigma_{0}}{\sqrt{3}(1+\eta) L}\left[1+\frac{2(1-2 \eta)^{2}}{(1+\eta)^{2}}\right]^{1 / 2} \cdot R_{0}^{2}\left[L+\frac{a \lambda}{2 \pi}-\frac{a \lambda}{2 \pi} \cos 2 N \pi\right] .
$$

\section{Energy dissipation due to friction}

The friction loss $W_{f}$ is given by

$$
W_{f}=\int_{S} \tau|\Delta v| \mathrm{d} s
$$

where $|\Delta v|$ is the velocity difference between platens and the cylinder in the direction of shear and parallel to the platen surface, i.e.,

$$
|\Delta v|=[(1-2 \eta) /(1+\eta)] \cdot(U R / L),
$$

and $\mathrm{ds}=2.2 \pi \mathrm{RdR}$.

From (1) and (8), after integration we can get

(i) For $\tau=\mu p_{a v}$,

$$
W_{f}=\frac{4 \pi(1-2 \eta) \mu U R_{0}^{3}}{3(1+\eta) L} \cdot p_{a v} .
$$

(ii) For $\tau=\mu\left[p_{a v}+\rho_{0} \phi_{0}\right]$,

$$
W_{f}=\frac{4 \pi(1-2 \eta) \mu U R_{0}^{3}}{3(1+\eta) L}\left[p_{a v}+\rho_{0} \phi_{0}\right] .
$$

(iii) For $\tau=\mu\left[p_{a v}+\rho_{0} \phi_{0}\left(1-\frac{R}{n R_{0}}\right)\right]$,

$$
W_{f}=\frac{4 \pi(1-2 \eta) \mu U R_{0}^{3}}{3(1+\eta) L}\left[p_{a v}+\rho_{0} \phi_{0}\left\{1-\frac{3}{4 n}\right\}\right] .
$$

(iv) For $\tau=\mu\left[p_{a v}+\rho_{0} \phi_{0}\left(1-\frac{R_{m}-R}{n R_{0}}\right)\right]$,

$$
W_{f}=\frac{4 \pi(1-2 \eta) \mu U R_{0}^{3}}{3(1+\eta) L}\left[p_{a v}+\rho_{0} \phi_{0}\left\{1-\frac{R_{m}}{n R_{0}}+\frac{3}{4 n}\right\}\right] .
$$




\section{Die load}

For plastic deformation of a metal-powder the external power ${ }^{*}$ supplied by the platens is given as

$$
\stackrel{*}{J}=W_{i}+W_{f}+W_{a}+W_{t} .
$$

The first term on the right hand side denotes the rate of internal energy dissipation $W_{i}$, the second term denotes the frictional shear energy losses $W_{f}$, the third term denotes the energy dissipation due to inertia forces, $W_{a}$, and the last term covers power supplied by predetermined body tractions, $W_{t}$. In this case forces due to inertia are negligibly small and no external surface traction is stipulated. Therefore, $W_{a}=W_{t}=0$.

Now the external power $J$ supplied by the press for the entire hexagonal shape through the platen is

$$
\stackrel{*}{J}=\int F_{i} U_{i} \mathrm{~d} s=P U, \quad \text { and }=P=p_{a v} \cdot 2 \pi R_{0} .
$$

(i) For $\tau=\mu p_{a v}$,

$$
\begin{aligned}
\frac{p_{a v}}{\sigma_{0}}= & {\left[1-\frac{2(1-2 \eta) \mu}{3(1+\eta) L} R_{0}^{2}\right]^{-1} \times \frac{2 \pi(1-2 \eta) U \sigma_{0}}{\sqrt{3}(1+\eta) L}\left[1+\frac{2(1-2 \eta)^{2}}{(1+\eta)^{2} L}\right]^{1 / 2} } \\
& \cdot R_{0}\left[L+\frac{a \lambda}{2 \pi}-\frac{a \lambda}{2 \pi} \cos 2 N \pi\right] .
\end{aligned}
$$

(ii) For $\tau=\mu\left[p_{a v}+\rho_{0} \phi_{0}\right]$,

$$
\begin{aligned}
\frac{p_{a v}}{\sigma_{0}}= & {\left[1-\frac{2(1-2 \eta) \mu}{3(1+\eta) L}\{1+x\} R_{0}^{2}\right]^{-1} \times \frac{2 \pi(1-2 \eta) U \sigma_{0}}{\sqrt{3}(1+\eta) L} } \\
& {\left[1+\frac{2(1-2 \eta)^{2}}{(1+\eta)^{2} L}\right]^{1 / 2} \cdot R_{0}\left[L+\frac{a \lambda}{2 \pi}-\frac{a \lambda}{2 \pi} \cos 2 N \pi\right] . }
\end{aligned}
$$

(iii) For $\tau=\mu\left[p_{a v}+\rho_{0} \phi_{0}\left(1-\left(R / n R_{0}\right)\right]\right.$,

$$
\begin{aligned}
\frac{p_{a v}}{\sigma_{0}}= & {\left[1-\frac{2(1-2 \eta) \mu}{3(1+\eta) L}\left\{1+x\left(1-\frac{3}{4 n}\right)\right\} R_{0}^{2}\right]^{-1} \times \frac{2 \pi(1-2 \eta) U \sigma_{0}}{\sqrt{3}(1+\eta) L} } \\
& {\left[1+\frac{2(1-2 \eta)^{2}}{(1+\eta)^{2} L}\right]^{1 / 2} \cdot R_{0}\left[L+\frac{a \lambda}{2 \pi}-\frac{a \lambda}{2 \pi} \cos 2 N \pi\right] }
\end{aligned}
$$

(iv) For $\tau=\mu\left[p_{a v}+\rho_{0} \phi_{0}\left(1-\left(R_{m}-R\right) / n R_{0}\right)\right]$,

$$
\begin{aligned}
\frac{p_{a v}}{\sigma_{0}}= & {\left[1-\frac{2(1-2 \eta) \mu}{3(1+\eta) L}\left\{1+x\left(1-\frac{R_{m}}{n R_{0}}+\frac{3}{4 n}\right)\right\} R_{0}^{2}\right]^{-1} } \\
& \times \frac{2 \pi(1-2 \eta) U \sigma_{0}}{\sqrt{3}(1+\eta) L}\left[1+\frac{2(1-2 \eta)^{2}}{(1+\eta)^{2} L}\right]^{1 / 2} \\
& \cdot R_{0}\left[L+\frac{a \lambda}{2 \pi}-\frac{a \lambda}{2 \pi} \cos 2 N \pi\right] .
\end{aligned}
$$




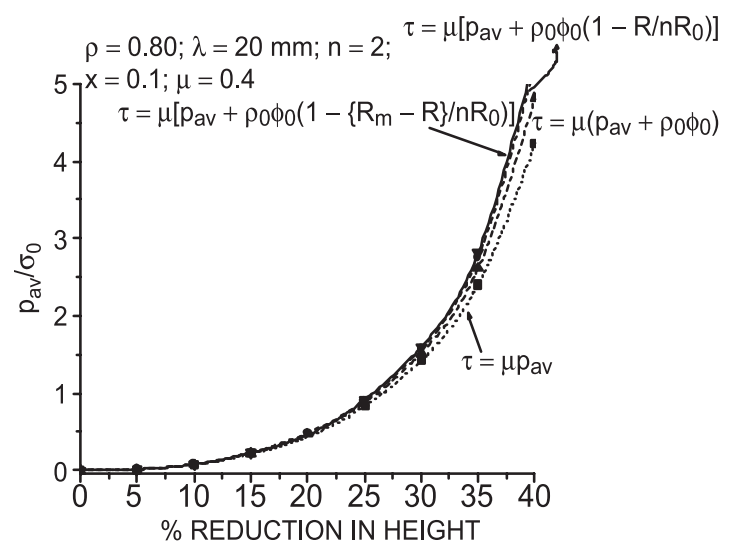

Figure 3. Variation of relative average forging pressure with percentage reduction in height of preform.

\section{Results and discussions}

Figure 3 shows the variation of relative average forging pressure with percentage reduction in height of the preform considering different friction laws. For lower values, of percentage reduction all the four laws give approximately the same result. But for higher reduction ratios, it can be distinguished. $\tau=\mu p_{a v}$ gives lower values of relative forging pressure and $\tau=\mu\left[p_{a v}+\rho_{0} \phi_{0}\left(1-\left(R / n R_{0}\right)\right)\right]$ gives higher values of relative forging pressure. Also, $\tau=\mu\left[p_{a v}+\rho_{0} \phi_{0}\left(1-\left(R_{m}-R\right) / n R_{0}\right)\right]$ gives a similar result as $\tau=\mu\left[p_{a v}+\rho_{0} \phi_{0}\left(1-R / n R_{0}\right)\right]$. Figure 4 shows that relative average forging pressure decreases with increasing slenderness ratio $(L / D)$. For higher values of $L / D$ there is no any difference among all the four interfacial friction laws. However for lower values of $L / D \tau=\mu\left[p_{a v}+\rho_{0} \phi_{0}\left(1-R / n R_{0}\right)\right]$ gives a higher relative pressure than $\tau=\mu\left[p_{a v}+\rho_{0} \phi_{0}\left(1-\left(R_{m}-R\right) / n R_{0}\right)\right]$. From figure 5 , it can be observed that relative average forging pressure increases on increasing the value of coefficient of friction. Again $\tau=\mu\left[p_{a v}+\rho_{0} \phi_{0}\left(1-R / n R_{0}\right)\right]$ gives a higher value of relative pressure and $\tau=\mu p_{a v}$ gives a lower value of relative pressure. From all the above graphs and results, it can be seen that the trend and order of variation of all the interfacial friction laws is the same. The decisive factors for selection of the better interfacial friction law are thus the percentage reduction in the height and slenderness ratio of the preform, and value of the coefficient of friction.

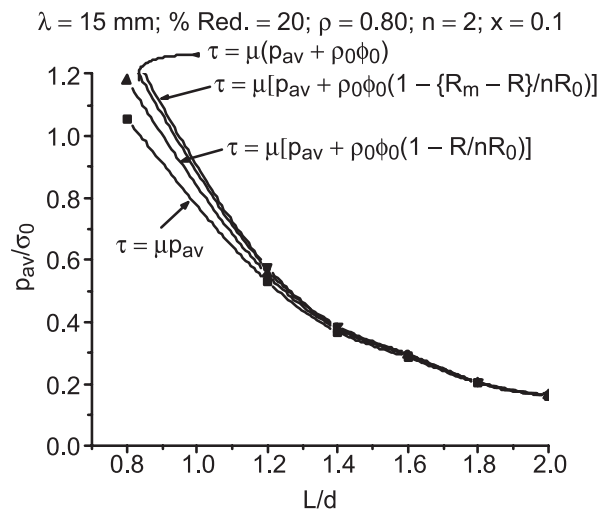

Figure 4. Variation of relative average forging pressure with slenderness ratio $(L / D)$. 


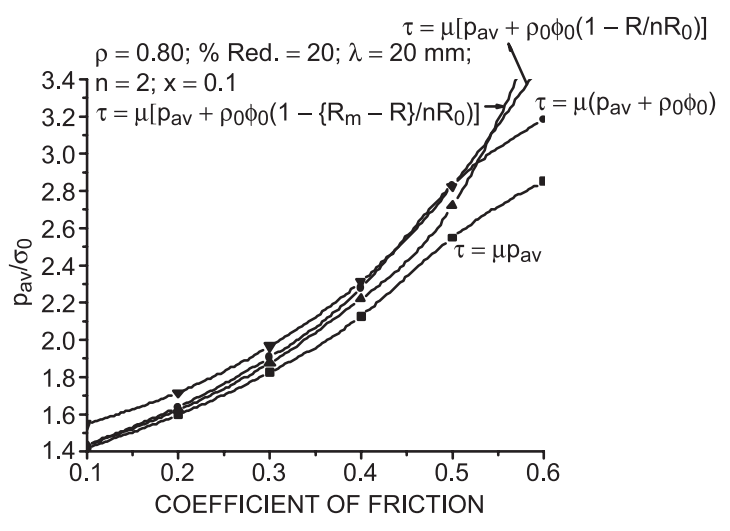

Figure 5. Variation of relative average forging pressure with coefficient of friction $(\mu)$.

\section{Conclusion}

Selection of interfacial friction law depends upon the size of the preform, percentage reduction in its height and coefficient of friction between die and preform. During the compression of a metal-powder preform, the compressive force gradually increases the relative density, which is directly proportional to the real area of contact. The real area of contact grows and approaches the apparent one as force increases, which in turn increases the relative density. It is observed that the formability of powder preforms improves with increase in the initial relative density. Since real area of contact gradually increases, assumption of friction behaviour as a constant one (i.e. $\tau=\mu p_{a v}$ ) is not correct. Also, the real area of contact depends upon the amount of force applied and during large deformation the sticking phenomenon cannot be ignored. Hence, before selecting an appropriate interfacial friction law, we should consider all these process parameters, which are involved in the deformation phase. This is why the most practical interfacial friction law is $\tau=\mu\left[p_{a v}+p_{0} \phi_{0}\left(1-\left(R_{m}-R\right) / n R_{0}\right)\right]$, which gives better results in practice. It is expected that these results will be helpful in analysing the forming problem.

\section{List of symbols}

$\begin{array}{ll}a & \text { amplitude; } \\ D & \text { diameter of the preform; } \\ J_{2}^{\prime} & \text { second invariant of deviatoric stress; } \\ k & \text { constant equal to } 2 \text { in yield criterion; } \\ L & N \lambda=\text { length of the preform; } \\ n & \text { constant quantity much greater than } 1 ; \\ p_{a v} & \text { ram pressure; } \\ R & \text { radius of the preform; } \\ R_{m} & \text { radius of the sticking zone; } \\ R, \theta, Y & \text { cylindrical co-ordinates; } \\ U & \text { ram velocity; } \\ \varepsilon_{R}, \varepsilon_{\theta}, \varepsilon_{y} & \text { principal strain increment; } \\ \eta & \text { constant and a function of } \rho \text { only; } \\ \lambda & \text { wavelength; }\end{array}$


$\mu \quad$ coefficient of friction;

$\rho$ relative density of the preform;

$\rho_{r} \quad$ real density of the preform;

$\sigma_{0} \quad$ yield stress of the non-work hardening matrix metal;

$\tau \quad$ shear stress.

\section{References}

Agrawal M, Kumar S, Jha A K 1999 High speed forging of hollow metal. J. Inst. Eng. (India) 80: 8-15 Deryagin B V 1952 What is friction? Izd. Akad. Nauk, USSR

Jha A K, Kumar S 1996 Dynamic effect during high speed sinter-forging process. Int. J. Mach. Tool Des. Res. 36: 1109-1113

Jha A K, Kumar S 1997 Investigation into high-speed forging of sintered copper powder strips. $J$. Mater. Process. Technol. 71: 394

Jha A K, Kumar S 1983 Interfacial friction during cold processing of metal powder preforms. Proc. Int. Tribology Conference, The Institutrion of Engineers, $\mathrm{p} 346$

Kumar S, Jha A K, Singh R K, Singh S 2001 Sintered preforms adds better value to aerospace components. J. Inst. Eng. (India) 82: 1-6

Ramakrishnan P 1980 Proc. Int. Seminar on Metal Working Technology Today and Tomorrow, Ranchi p. 43

Rooks B W 1974 The effect of die temperature on metal flow and die wear during high speed hot forging. 15th Int. MTDR. Conference, Birmingham, U K p. 487

Sutradhar G, Jha A K, Kumar S 1995 Cold forging of sintered iron-powder preforms. J. Mater. Proc. Technol. 52: 369-386

Tabata T, Masaki S 1975 Plane strain extrusion of porous materials. J. Jpn. Soc. Technol. Plasticity 16: 279-284

Tabata T, Masaki S, Abe Y 1977 A yield criterion for porous metals and analysis of axial compression of porous discs. J. Jpn. Soc. Technol. Plast. 18: 373-380 